\title{
Back from the brink: retrieval of membrane proteins from terminal compartments.
}

\author{
Matthew N.J. Seaman \\ University of Cambridge, Cambridge Institute for Medical \\ Research, Wellcome Trust/MRC Building, Addenbrookes \\ Hospital, CB2 OXY, UK.
}

Contact: mnjs100@cam.ac.uk, 44-1223-763225.

\section{Subtitle}

Unexpected pathways for membrane protein retrieval from vacuoles and endolysosomes.

Keywords: lysosome; recycling; retrieval; vacuole, Snx4p, AP5 complex

\section{Summary}

It has long been believed that membrane proteins present in degradative compartments such as endolysosomes or vacuoles would be destined for destruction. Now however, it appears that mechanisms and machinery exist in simple eukaryotes such as yeast and more complex organisms such as mammals that can rescue potentially 'doomed' membrane proteins by retrieving them from these 'late' compartments and recycling them back to the Golgi complex. In yeast, a sorting nexin dimer containing Snx4p can recognise and retrieve the Atg27p membrane protein whilst in mammals, the AP5 complex (with SPG11 and SPG15) directs the recycling of Golgi-localised proteins along with the cation-independent mannose 6-phosphate receptor (CIMPR). Although the respective machinery is different, there is much commonality between yeast and mammals regarding the mechanisms of retrieval and the physiological importance of these late recycling pathways. 


\section{Introduction}

One of the defining features of eukaryotic cells is the system of discrete membrane-bound organelles present in the cytoplasm that comprise the secretory and endocytic pathways. These compartments enable the cell to take up nutrients from its environment and sense changes in that environment along with secreting small molecules that may be necessary for communication between cells in a multicellular organism. Biochemically competing reactions such as synthesis and degradation can be spatially separated whilst the movement of a protein along the secretory pathway can allow for sequential modification or processing of certain proteins in a manner akin to a production line in a factory. The different organelles of the endocytic and secretory pathways are populated with membrane proteins that confer a specific function and identity to the various organelles and therefore the mechanisms that control the movement and exchange of membrane proteins between the different organelles need to be highly sophisticated and efficient to ensure the correct membrane protein is delivered to the appropriate organelle. This process is known as membrane trafficking and the mechanisms and principles of membrane trafficking are generally very well conserved through evolution (for review see ${ }^{[1]}$ ) - see Figure 1.

A structure that is conserved in evolution and present in the cells of complex organisms such as humans, or simple organisms such as yeast is a degradative organelle respectively called the lysosome or vacuole. This is sometimes referred to as the 'stomach' or 'trash-can' of the cell as proteins delivered to the lysosome/vacuole are degraded by hydrolytic enzymes and the amino acids released used for new protein synthesis (reviewed in [2,3]). The lysosome/vacuole lies at the end of the endocytic pathway and has, for many years, been regarded as the end of the line - literally. Proteins delivered to a lysosome/vacuole could not leave but instead would be destroyed. To ensure that only proteins that are 'disposable' are delivered to the lysosome/vacuole, cells have the means to sort and recycle proteins from the endosome and direct those proteins back to either the Golgi complex or to the plasma membrane (cell surface). A good example of membrane protein recycling is provided by the 
receptors that actually sort the hydrolytic enzymes (e.g. proteases and lipases) that function as the engines of destruction in the lysosome/vacuole. In mammalian cells, a membrane protein called the mannose 6-phosphate receptor (MPR) binds to inert lysosomal hydrolases in the Golgi complex and directs them into vesicles for delivery to the lysosome via an endosome. When the MPR arrives, with its protease ligand, at an endosome, the ligand falls off the MPR which is then recycled back to the Golgi by a set of proteins called retromer $[4,5]$. The hydrolase continues on its journey to the lysosome/vacuole where, upon arrival, it will be activated. The process is virtually identical in a simple eukaryote such as yeast but the yeast counterpart of the MPR is a protein called Vps10p [6-8]. Thus the MPR or Vps10 protein can undertake multiple rounds of protease sorting to ensure that the lysosome/vacuole is always well stocked with hydrolases for degrading whatever arrives there [9].

Interestingly, it has emerged recently that, in yeast, it is possible for a membrane protein to be recycled from the vacuolar membrane. In mammalian cells too, there are mechanisms that can sort and recycle proteins from structures that are difficult to distinguish from lysosomes. Thus the long accepted notion that the lysosome/vacuole is the end of the line where proteins go to die is no longer strictly true. In this article, I will describe the recently published data that has led to the realisation that for some proteins, there is a comeback from what was previously regarded as a 'no return' or 'dead-end' destination.

\section{Retrieval from the yeast vacuole}

Studies in yeast have recently revealed that a membrane protein called Atg27p can be recycled from the vacuolar membrane back to the Golgi. This recycling pathway actually involves two steps; firstly retrieval from the vacuole to an endosome and then recycling from an endosome to the Golgi [10]. The Atg27 protein is a type-I (single pass) membrane protein that has been implicated in the process of autophagy in yeast [11] - for a brief summary of autophagy, see Figure 2.

The Atg27 protein is important in regulating the trafficking of another protein called Atg9p that is necessary for autophagy. Atg27p functions in the sorting of 
Atg9p into vesicles that bud from the Golgi complex [12,13]. Atg9p is conserved in evolution but Atg27p is not present in all organisms being absent in higher eukaryotes such as mammals. Intriguingly however, the Atg27 protein has a structural fold that is present in MPR proteins suggesting perhaps that, in mammals, the MPR may be able to perform the function that is tasked to Atg27p in yeast. Interestingly in mammalian cells, the ATG9a protein and the cationindependent MPR display a similar localisation when autophagy is induced [14].

The retrieval of Atg27p from the vacuolar membrane requires the yeast Snx4 protein $[10,15]$. This is a cytoplasmic protein that can peripherally associate with the vacuolar membrane due to the presence of a p40 phox homology (PX) domain in the Snx4 protein. The PX domain, like Snx4, is conserved in evolution and is a defining feature of a family of proteins called sorting nexins abbreviated to Snx - and can mediate membrane binding to phosphatidylinositol 3-phosphate (PtdIns3P) or sometimes phosphatidylinositol 3,5-bisphosphate (PtdIns3,5P $)^{[16,17] . ~}$

\section{The sorting nexin connection}

Sorting nexins comprise a somewhat diverse family of proteins that, through their PX domain, are associated with endosomal compartments and perform a range of tasks related to membrane trafficking or signalling depending on what other functional domains are present in the respective sorting nexin [18,19]. The retromer complex that is required for MPR or Vps10p recycling from endosomes to the Golgi contains a dimer of Snx proteins; Vps5p and Vps17p in yeast and SNX1 or SNX2 with either SNX5 or SNX6 in mammals [8,20,21]. For Atg27p retrieval from the vacuole, Snx4p interacts with the cytoplasmic tail of Atg27p and thereby sequesters the protein in a specific domain of the vacuolar membrane. Another feature present in some sorting nexin proteins is a Bin-AmphiphysinRvs (BAR) domain that can drive the formation of membrane tubules [22] and Snx4p does indeed possess a BAR domain. The truncation of Snx4p to delete either the PX or the BAR domain results in a non-functional protein that cannot mediate the retrieval of Atg27p from the vacuole. The details of the Snx $4 p$ Atg27p interaction have yet to be worked out but residues in the cytoplasmic tail 
of Atg27p close to the trans-membrane domain are necessary for Atg27p retrieval and association with $\operatorname{Snx} 4 \mathrm{p}$ [10].

This is not, however, the first report that a sorting nexin protein can mediate the selection of membrane proteins ('cargo') for trafficking from one compartment to another. It has been previously reported that mammalian SNX1 and SNX2 can associate with receptor tyrosine kinases such as the epidermal growth factor receptor (EGFR) and the insulin receptor (IR) and have been reported to bind to the cytoplasmic tail of the cation-independent MPR (CIMPR) [23-26]. Additionally, the SNX3 protein (known as Grd19p in yeast), a sorting nexin that contains a PX domain and little else, can interact with membrane proteins to mediate their sorting into transport intermediates [27].

For retromer-mediated recycling from endosomes, the sorting nexin component is generally a heterodimer as BAR domains have to dimerize in order to drive tubule formation. The retrieval of Atg27p from the vacuole requires Snx4 to associate with another sorting nexin protein, either Snx41p or Snx42p. These sorting nexin proteins may be functionally redundant in Atg27p trafficking from the vacuole although there is some discrepancy within the literature as to whether Snx41p is required for Atg27p retrieval [10,15].

\section{Regulating lipid content and protein localisation through $S n \times 4 p$}

In addition to functioning in the retrieval of Atg27p from the vacuolar membrane, it has recently been reported that Snx4p (along with Snx42p) also plays a key role in regulating the lipid content of endosomes and the vacuole [28]. Cells lacking Snx4p display an autophagy defect that is markedly exacerbated by inhibition of phosphatidylserine (PtdSer) synthesis at mitochondria. In the absence of Snx4p, PtdSer accumulates in endosomes and leads to impaired induction of autophagy and fusion of autophagosomes with vacuoles. Snx $4 \mathrm{p} / \mathrm{Sn} \times 42 \mathrm{p}$ dimers demonstrated preferential binding to membranes enriched in PtdSer suggesting that a function of $\operatorname{Snx} 4 \mathrm{p}$ may be to export PtdSercontaining membranes from endosomes. Mechanistically, it is not yet clear if Snx4p sorts PtdSer in a manner similar to how it sorts Atg27p so there are 
important details yet to be elucidated with respect to $\operatorname{Snx} 4 \mathrm{p}$ involvement in regulating the lipid content of endosomes.

Interestingly, studies published some years ago established Snx4p in yeast as an important protein in the endosome-to-Golgi recycling of a membrane protein called Snc1p - a member of the SNARE family of proteins that mediate fusion of vesicles with their target organelle [29]. It has now been shown that Snx4p involvement in Snc1p recycling requires Snx42p (also known as Atg20p) but not Snx41p [15]. Thus it may be that Snx4p forms distinct complexes with either Snx41 or Snx42 according to which membrane protein it is acting to retrieve, Atg27p or Snc1p. The different Snx4p heterodimers may also confer distinct preferences for the site of action, vacuolar or endosomal membranes respectively through interactions with both cargo molecules and phosphatidylinositol phosphates in a manner akin to coincidence detection - the process the underpins the targeting of many other vesicle coats to their respective site of action. Following Snx4-mediated retrieval of Atg27p from the vacuolar membrane to the endosome, Atg27p is then recycled back to the Golgi by the retromer complex. It has yet to be determined how retromer mediates the retrieval of $\operatorname{Atg} 27 \mathrm{p}$ but presumably a component of the retromer complex can bind to Atg27p and sort it into a vesicle or tubule for delivery to the Golgi complex.

It may be regarded as surprising that a membrane protein can be retrieved from a structure such as a vacuole that is full of hydrolytic enzymes as there is an obvious risk that some of the active proteases (and lipases) may be transferred from the vacuole to an endosome or back to the Golgi thereby causing a mixing of biosynthetic and degradative reactions that the endomembrane system of a eukaryotic cell is meant to segregate. It should be noted however that the tubules generated by SNX-BAR protein (e.g. Snx4p) assembly are well suited to retrieval of membrane proteins as they have a high surface area to volume ratio and can therefore concentrate a lot of membrane proteins whilst excluding the soluble content of the organelle from which the tubule is formed (for review see [30]).

\section{New machinery for an old pathway?}


Although I said at the beginning of this essay that it had been believed that the vacuole is a 'dead-end' for membrane proteins, there have been tantalising clues that it may be possible for a membrane protein to be recycled from the vacuole. Previous studies from $\sim 20$ years ago indicated that a reporter protein containing both the functional domain of the vacuolar alkaline phosphatase protein and the cytoplasmic tail of the Golgi resident endopeptidase, dipepidyl aminopeptidase A (DPAPA) could recycle from the yeast vacuole to the Golgi [31]. In that instance, Vac7p, a yeast protein that localises to the vacuole and is required for vacuolar inheritance, was required for the DPAPA-ALP fusion protein to be recycled. Vac7p can regulate levels of PtdIns3,5 $\mathrm{P}_{2}$ on the vacuolar membrane [32] but it is not yet known whether Vac7p influences the activity of Snx4p in Atg27p recycling (through modulating PtdIns3,5 $\mathrm{P}_{2}$ levels) or if $\mathrm{Snx} 4 \mathrm{p}$ is required for the recycling of the DPAPA-ALP fusion protein, i.e. is the pathway and machinery for recycling of Atg27p the same as that for DPAPA-ALP?

\section{Retrieval from endolysosomes in mammalian cells}

The ability to retrieve a membrane protein from an organelle such as a vacuole is not restricted only to yeast. In mammalian cells however, the sorting nexin protein SNX4, although conserved, functions to direct the endosome-to-cell surface retrieval of the transferrin receptor and has not been reported to have a role in membrane trafficking from compartments such as the lysosome that is the functional equivalent of the yeast vacuole [33].

Recent data obtained from mammalian cells shows that proteins such as the MPR can be retrieved from compartments called endolysosomes that bare many similarities to lysosomes - see Figure 3. In this instance, retrieval is mediated the AP5 complex, a heterotetrameric protein complex that belongs to the family of clathrin adaptor proteins and is related to the clathrin adaptors AP1 and AP2 that respectively function at the trans-Golgi network (TGN) and the plasma membrane [34,35]. The AP5 complex was first identified a few years ago as an evolutionary ancient protein complex that localises to LAMP1 (lysosomeassociated membrane glycoprotein-1)-positive organelles in mammals. These are compartments that are considered to be very late in the endocytic pathway 
[36]. In fact, LAMP1 is also regarded as a marker for lysosomes. Unlike AP1 and AP2, the AP5 complex does not associate with clathrin but rather with two proteins called SPG11 and SPG15 - both of which, when mutated, can cause the movement disorder, hereditary spastic paraplegia (HSP). The SPG11 and SPG15 proteins share some structural features present in coat proteins such as clathrin and others coats like the COPI coat present at the Golgi and the COPII coat on the endoplasmic reticulum (ER) and may drive vesicle formation like the COPI and COPII coats (see Figure 4). The SPG15 protein also has a FYVE (Fab1-YOTB-Vac1EEA1) domain that can bind to PtdIns3P - a lipid that is required for the localisation and/or recruitment of the AP5-SPG11/15 machinery to the endolysosome.

\section{Cargo recognition by the AP5-SPG11/15 machinery}

The recent advance in understanding the function of the AP5 complex came through using a mass spectrometry-based proteomic analysis of vesicles from cells lacking AP5 function to identify which membrane proteins depend on AP5 for their localisation [34]. One of the proteins identified was the MPR, specifically the cation-independent mannose 6-phosphate receptor (CIMPR) which is known to cycle from the TGN to the endosome using clathrin-based sorting machinery and then back to the TGN by retromer-mediated recycling. In addition to the CIMPR were other membrane proteins that localise to the Golgi at steady state but presumably exit the Golgi (possibly at a low rate) and then are retrieved by the AP5-SPG11/15 machinery.

The recognition of cargo proteins such as the CIMPR by the AP5-SPG11/15 machinery most likely occurs through mechanisms distinct from other adaptor protein complex like AP1 or AP2 where the medium $(\mu)$ subunits bind to tyrosine-based sorting motifs that conform to the consensus of $\operatorname{Yx} \Phi$ (where $\Phi$ is a bulky hydrophobic residue). This is because the $\mu 5$ subunit of AP5 lacks the necessary amino acids to form an interaction with $\operatorname{Yxx} \Phi$ motifs although it is possible that the $\mu 5$ subunit might interact with motifs distinct from the canonical $\operatorname{Yx} \Phi$ motif [36]. In fact, given that many proteins present in endolysosomes (e.g. LAMP1) have $\mathrm{Yxx} \Phi$ motifs, the AP5 complex would 
recognise those proteins as cargo if it were able to bind $\mathrm{Yx} \Phi$ motifs but this currently does not appear to be the case. Through in-vitro experiments, in has been reported however that the SPG15 protein can associate with the CIMPR [34] although, at this time, the precise mechanisms that govern the interaction between AP5-SPG11/15 and cargo remain to be determined.

\section{A back-up pathway for retromer?}

Interestingly it appears that the AP5-mediated retrieval of proteins such as the CIMPR occurs from organelles that would be considered to be much 'later' along the endocytic pathway than the endosomes where retromer operates. Indeed it may be that AP5 exists to capture and recycle membrane proteins that have somehow escaped recycling by retromer. Loss of AP5 causes the CIMPR to become mislocalised although not as much as the loss of retromer function. However, loss of both AP5 and retromer has an additive effect and as AP5 is present on LAMP1-positive endolysosomes, the hypothesis that AP5 is there to 'clear up' after retromer is plausible although it is also possible that AP5 drives the retrieval of proteins from endolysosomes irrespective of retromer function. Possibly AP5 now exists as a back-up mechanism to ensure that retrieval of proteins such as the CIMPR is as efficient as possible and it has been shown that AP5 expression is very low relative to other adaptor complexes such as AP1 or AP2 suggesting that it is not required to sort as many membrane proteins as machinery that is more highly expressed such as the retromer complex. In fact AP5 is not ubiquitously conserved having been lost from many organisms including yeast.

\section{Hereditary spastic paraplegia and the endosome connection}

As mentioned previously, the SPG11 and SPG15 proteins that operate with the AP5 complex are linked to a neurodegenerative disease, namely hereditary spastic paraplegia (HSP), a length-dependent axonopathy that causes loss of long motor neurons and leads to a progressive spastic paralysis of the lower limbs. In the case of patients with SPG11 or SPG15 mutations, there are additional 
complications that result in a more severe neurological phenotype. It's not yet known precisely how diseases such as HSP cause neurodegeneration but it has been observed that cells with SPG11 or SPG15 mutations have aberrant lysosomes that accumulate material that is normally broken down in the lysosome [37] and several SPG genes which, when mutated cause HSP, encode proteins that operate in endosomal protein sorting. For example, Strumpellin, a protein encoded by the SPG8 gene, which functions as part of the WASH complex that associates with retromer [38]. It has also been reported that loss of SPG15 function can impair the reformation of lysosomes from autolysosomes - a process that is likely to be mechanistically similar to the reformation of lysosomes from endolysosomes. - resulting in defects to autophagy [39-41].

Thus, both yeast and mammalian cells have mechanisms and machinery that can retrieve membrane proteins from vacuoles or endolysosomes but the mechanisms are different in the two eukaryotic cells. Although AP5 (along with SPG11 and SPG15) is an evolutionary ancient set of machinery, yeast do not have an AP5 complex (or homologues of the SPG11/15 proteins) [36]. Generally the machinery that mediates membrane trafficking is very highly conserved in eukaryotes so this is something of an exception. One possible reason why yeast may have lost AP5 is that the vacuole in yeast exists as either one or a very small number of relatively large structures in the cell and, to date, there is no evidence for much heterogeneity within the small population of vacuoles in yeast. In mammals however it has been shown that organelles with all the attributes of a lysosome (e.g. positive for LAMP1 and hydrolases such as cathepsin D) are not necessarily active and may not be acidic [42]. Whereas, organelles that are described as endolysosomes by virtue of having properties of both endosomes (e.g. positive for the MPR) and lysosomes (acidic $\mathrm{pH}$ ) have been shown to be proteolytically active in a manner expected for a bona fide lysosome. Additional factors such as the position of the endolysosome or lysosome in a mammalian cell can also influence its function [43]. Alternatively, the loss of AP5 in evolution could indicate that, for many organisms, retromer-mediated endosome-to-Golgi retrieval is sufficiently efficient to maintain an active pool of proteins such as the CIMPR at the Golgi complex thus rendering AP5 redundant. 


\section{Conclusions}

The boundaries that have defined endosomes and lysosomes as totally distinct organelles are becoming somewhat blurred. From the studies on AP5, it is possible to propose that the retrieval of membrane proteins from endolysosomes is mediated by AP5. In yeast however, there is no clear equivalent to the endolysosome and perhaps a need for different machinery has driven the adoption of Snx $4 \mathrm{p}$ as the key machinery for retrieval from vacuoles. In the case of yeast, $\operatorname{Snx} 4 \mathrm{p}$ functioning as a heterodimer with either $\operatorname{Snx} 41 \mathrm{p}$ or Snx $42 p$ provides the means to recycle proteins such as Atg27p from apparently 'late' structures such as the vacuole or from earlier structures such as an endosome for the recycling of the Snc1 proteins. A feature of these trafficking pathways, be it the Snx4p pathway in yeast, or the AP5 pathway in mammals, that is conserved is the importance of the role of lipids, especially the phosphatidylinositol phosphates. Both sets of machinery rely on PtdIns3P (and possibly PtdIns 3,5 $\mathrm{P}_{2}$ ) for their respective membrane associations and thus the mechanisms that govern the production and turnover of PtdIns3P will play a key role in regulating the activity of the two sets of machinery. It is also interesting that both the AP5-SPG11/15 pathway, and the Snx4p-mediated retrieval of Atg27p have implications for the recovery of membrane or proteins important in autophagy. Therefore, although different machineries are involved, yeast and mammalian cells demonstrate significant commonality when it comes to retrieval from these 'end-of-the-road' compartments (see Figure 5).

There is much yet to learn about how pathways that sort membrane proteins for recycling from vacuolar or endolysosomal compartments function at the mechanistic and molecular level but, as research advances the understanding of how proteins are recycled from vacuoles or endolysosomes, there is now a growing appreciation of the physiological importance of these pathways that are very much the 'last chance' for membrane proteins in the endocytic system.

\section{Acknowledgements}


I would like to thank Dr Jenny Hirst for critical reading of this article and many helpful suggestions.

\section{References}

1. J.B. Dacks, M.C. Field, Curr Opin Cell Biol. 2018 53, 70.

2. R.M Perera, R. Zoncu, Annu Rev Cell Dev Biol. 2016 32, 223.

3. L. Wartosch N.A. Bright, J.P. Luzio, Curr Biol. 2015 25, R315. doi: 10.1016/j.cub.2015.02.027.

4. M.N. Seaman, J Cell Biol. 2004 165, 111.

5. C. N. Arighi, L. M. Hartnell, R. C. Aguilar, C. R. Haft, J. S. Bonifacino, J. Cell Biol. 2004, 165, 123.

6. E.G. Marcusson, B.F. Horazdovsky, J.L. Cereghino, E. Gharakhanian, S.D. Emr, Cell 1994, 77, 579.

7. M.N. Seaman, E.G. Marcusson, J.L. Cereghino, S.D. Emr. (1997). J Cell Biol. 1997, 137, 79.

8. M.N. Seaman, J.M. McCaffery, S.D. Emr. J Cell Biol. 1998, 142, 665.

9. M.N. Seaman, Trends Cell Biol. 2005, 15, 68.

10. S.W. Suzuki, S.D. Emr, J Cell Biol. 2018, 217, 1623.

11. W.J. Yen, J.E. Legakis, U. Nair, D.J. Klionsky, Mol Biol Cell. 2007, 18, 581

12. S.K. Backues, D.P. Orban, A. Bernard, K. Singh, Y. Cao, D.J. Klionsky, Traffic. 2015, 16, 172.

13. V.A. Segarra, D.R. Boettner, S.K. Lemmon, Traffic. 2015 16, 365.

14. A.R. Young, E.Y. Chan, X.W. Hu, R. Köchl, S.G. Crawshaw, S. High, D.W. Hailey, J. Lippincott-Schwartz, S.A. Tooze. J Cell Sci. 2006, 119, 3888.

15. M. Ma, C.G. Burd, R.J. Chi, Traffic. 2017 18, 134.

16. M.L. Cheever, T.K. Sato, T. de Beer, T.G. Kutateladze, S.D. Emr, M. Overduin, Nat Cell Biol. 2001, 3, 613

17. J.W. Yu, M.A. Lemmon, J Biol Chem. 2001, 276, 44179.

18. J.G. Carlton, P.J. Cullen. Curr Biol. 2005, 15, 819.

19. L.F. Seet, W. Hong. Biochim Biophys Acta, 2006, 1761, 878.

20. B.F. Horazdovsky, B.A. Davies, M.N. Seaman, S.A. McLaughlin, S. Yoon, S.D. Emr. Mol Biol Cell. 1997, 8, 1529

21. T. Wassmer, N. Attar, M.V. Bujny, J. Oakley, C.J. Traer, P.J. Cullen. J Cell Sci. 2007, 120, 45.

22. B.J. Peter, H.M. Kent, I.G. Mills, Y. Vallis, P.J. Butler, P.R. Evans, H.T. McMahon. Science. 2004, 303, 495.

23. R.C. Kurten, D.L. Cadena, G.N. Gill. Science. 1996, 272, 1008.

24. C.R. Haft, M. de la Luz Sierra, V.A. Barr D.H. ,Haft, S.I. Taylor. Mol Cell Biol. 1998, 18, 7278.

25. B. Simonetti, C.M. Danson, K.J. Heesom, P.J. Cullen. J Cell Biol. 2017, 216, 3695.

26. A. Kvainickas, A. Jimenez-Orgaz, H. Nägele, Z. Hu, J. Dengjel, F. Steinberg. J Cell Biol. 2017, 216, 3677.

27. T.I. Strochlic, T.G. Setty, A. Sitaram, C.G. Burd. J Cell Biol. 2007, 177, 115.

28. M. Ma, S. Kumar, L. Purushothaman, M. Babst, C. Ungermann, R.J. Chi, C.G. Burd. Mol Biol Cell. 2018, mbcE17120743. doi: 10.1091/mbc.E17-12- 
0743.

29. E.H. Hettema, M.J. Lewis, M.W. Black, H.R. Pelham . EMBO J. 2003, 22, 548.

30. J.R. van Weering, P.J. Cullen. Semin Cell Dev Biol. 2014, 31, 40.

31. N.J. Bryant, R.C. Piper, L.S. Weisman, T.H. Stevens. J Cell Biol. 1998, 142, 651.

32. J.D. Gary, T.K. Sato, C.J. Stefan, C.J. Bonangelino, L.S. Weisman, S.D. Emr. Mol Biol Cell. 2002, 13, 1238.

33. C.J. Traer, A.C. Rutherford, K.J. Palmer, T. Wassmer, J. Oakley, N. Attar, J.G. Carlton, J. Kremerskothen, D.J. Stephens, P.J. Cullen. Nat Cell Biol. 2007, 9, 1370.

34. J. Hirst, D.N. Itzhak, R. Antrobus, G.H.H. Borner, M.S. Robinson. PLoS Biol. 2018, 16, e2004411. doi: 10.1371/journal.pbio.2004411.

35. J. Hirst, C. Irving, G.H. Borner. Traffic. 2013, 14, 153.

36. J. Hirst, L.D. Barlow, G.C. Francisco, D.A. Sahlender, M.N. Seaman, J.B. Dacks, M.S. Robinson. PLoS Biol. 2011, 9, e1001170. doi: 10.1371/journal.pbio.1001170.

37. J. Hirst, J.R. Edgar, T. Esteves, F. Darios, M. Madeo, J. Chang, R.H. Roda, A. Dürr, M. Anheim, C. Gellera, J. Li, S. Züchner, C. Mariotti, G. Stevanin, C. Blackstone, M.C. Kruer, M.S. Robinson. Hum Mol Genet. 2015, 24, 4984.

38. M.E. Harbour, S.Y. Breusegem, R. Antrobus, C. Freeman, E. Reid, M.N. Seaman. J Cell Sci. 2010, 123, 3703.

39. J. Chang, S. Lee, C. Blackstone. J Clin Invest. 2014, 124, 5249.

40. C. Vantaggiato, E. Clementi, M.T. Bassi. Autophagy. 2014, 10, 374.

41. R.E. Varga, M. Khundadze, M. Damme, S. Nietzsche, B. Hoffmann, T. Stauber, N. Koch, J.C. Hennings, P. Franzka, A.K. Huebner, M.M. Kessels, C. Biskup, T.J. Jentsch, B. Qualmann, T. Braulke, I. Kurth, C. Beetz, C.A. Hübner. PLoS Genet. 2015, 11, e1005454.

42. N.A. Bright, L.J. Davis, J.P. Luzio. Curr Biol. 2016, 26, 2233.

43. J.S. Bonifacino, J. Neefjes. Curr Opin Cell Biol. 2017, 47, 1.

44. C.F. Bento, M. Renna, G. Ghislat, C. Puri, A. Ashkenazi, M. Vicinanza, F.M. Menzies, D.C. Rubinsztein. Annu Rev Biochem. 2016, 85, 685.

45. F.M. Menzies, A. Fleming, A. Caricasole, C.F. Bento, S.P. Andrews, A. Ashkenazi, J. Füllgrabe, A. Jackson, M. Jimenez Sanchez, C. Karabiyik, F. Licitra, A. Lopez Ramirez, M. Pavel, C. Puri, M. Renna, T. Ricketts, L. Schlotawa, M. Vicinanza, H. Won, Y. Zhu, J. Skidmore, D.C. Rubinsztein. Neuron. 2017, 93, 1015.

46. J. Hirst, A. Schlacht, J.P. Norcott, D. Traynor, G. Bloomfield, R. Antrobus, R.R. Kay, J.B. Dacks, M.S. Robinson. elife, 2014, e02866, doi:10.7554/eLife.02886

\section{Figure legends}

Figure 1. The endocytic pathway.

In yeast and mammals the endocytic pathway functions similarly with delivery of proteins and lipids from the cell surface to an endosome. Once at the endosome, a protein can be sorted for delivery to the Golgi via a retrieval pathway, or recycled back to the cell surface - a process that involves retrieval to 
the Golgi in yeast. Proteins destined for the lysosome or vacuole have traditionally been regarded as heading for destruction as there was no known mechanisms or machinery to retrieve a protein from a lysosome or the vacuole.

Figure 2. Autophagy.

Autophagy is an evolutionary conserved survival mechanism that involves the sequestering of cytoplasmic proteins (or larger structures such as organelles within a double membrane-bound structure called the phagophore to produce an enclosed discrete entity called an autophagosome. The autophagosome then fuses with the lysosome/vacuole thereby gaining proteolytic enzymes that can digest the contents of the autophagosome. Thus the cell can turnover some of its cytoplasmic proteins to release amino acids for the synthesis of essential proteins and the process of autophagy therefore often operates under starvation conditions when nutrients are scarce (for review see ${ }^{[44]}$ ).

Autophagy is also important for the clearance of protein aggregates and damaged organelles and has been linked to several neurodegenerative diseases such as Parkinson's disease and Huntington's disease (reviewed in [45]).

Figure 3. The endolysosome.

The endolysosome is believed to be the product of fusion of an endosome with a lysosome. The resulting hybrid organelle has been shown to be acidic and stockedwith active proteases and therefore capable of degradative functions.

The process of reforming the lysosome may involve recycling membrane and proteins (e.g. the CIMPR) that are not destined for degradation and could occur via the AP5 pathway.

Figure 4. The AP5-SPG11/15 machinery.

A schematic diagram of the AP5 complex comprising the large 'adaptins' $\beta 5$ and $\zeta$ with the smaller subunits $\mu 5$ and $\sigma 5$. The SPG15 and SPG11 proteins may provide the self-assembly activity analogous to clathrin and may also be involved in selecting cargo proteins. The arrangement of the AP5 subunits is based upon protein association studies and on predictions from phylogenetic analyses of the sequences of the AP5 subunits [36, 46]. 
Figure 5. Schematic diagram of retrieval from 'terminal' compartments.

Generally, the mechanisms that govern membrane trafficking in eukaryotes are well conserved. For example, the AP1 complex (along with clathrin) is required to sort receptors (e.g. Vps10p or the CIMPR) and hyrolases into vesicles at the late-Golgi or trans-Golgi network. The endosome is a conserved and key structure in both simple eukaryotes such as yeast and complex metazoans such as mammals. At the endosome, the retromer complex mediates endosome-toGolgi retrieval in both yeast and mammals. In yeast, Snx4p can direct the vacuole to endosome retrieval of the Atg27 protein. In mammals, the AP5 complex with SPG11 and SPG15 mediate retrieval from 'late' or terminal structures such as endolysosomes and thereby act as a back-up mechanism for the retromer complex. Both Snx4p- and AP5-mediated protein recycling occurs from structures once considered to be only degradative organelles from where proteins could not escape intact. 


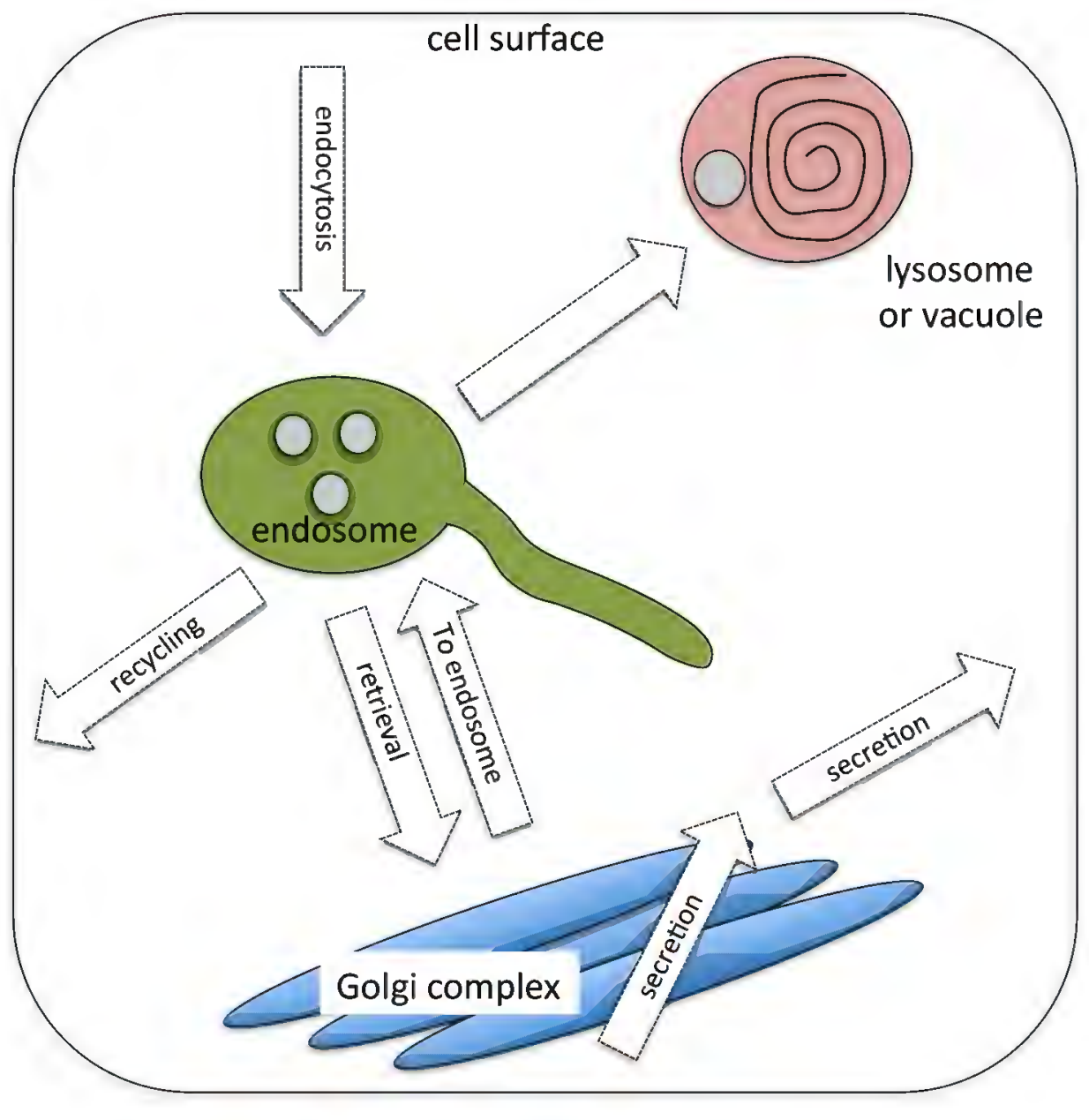


phagophore

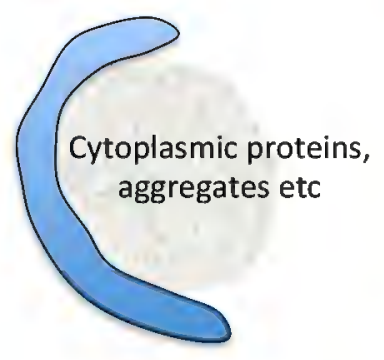

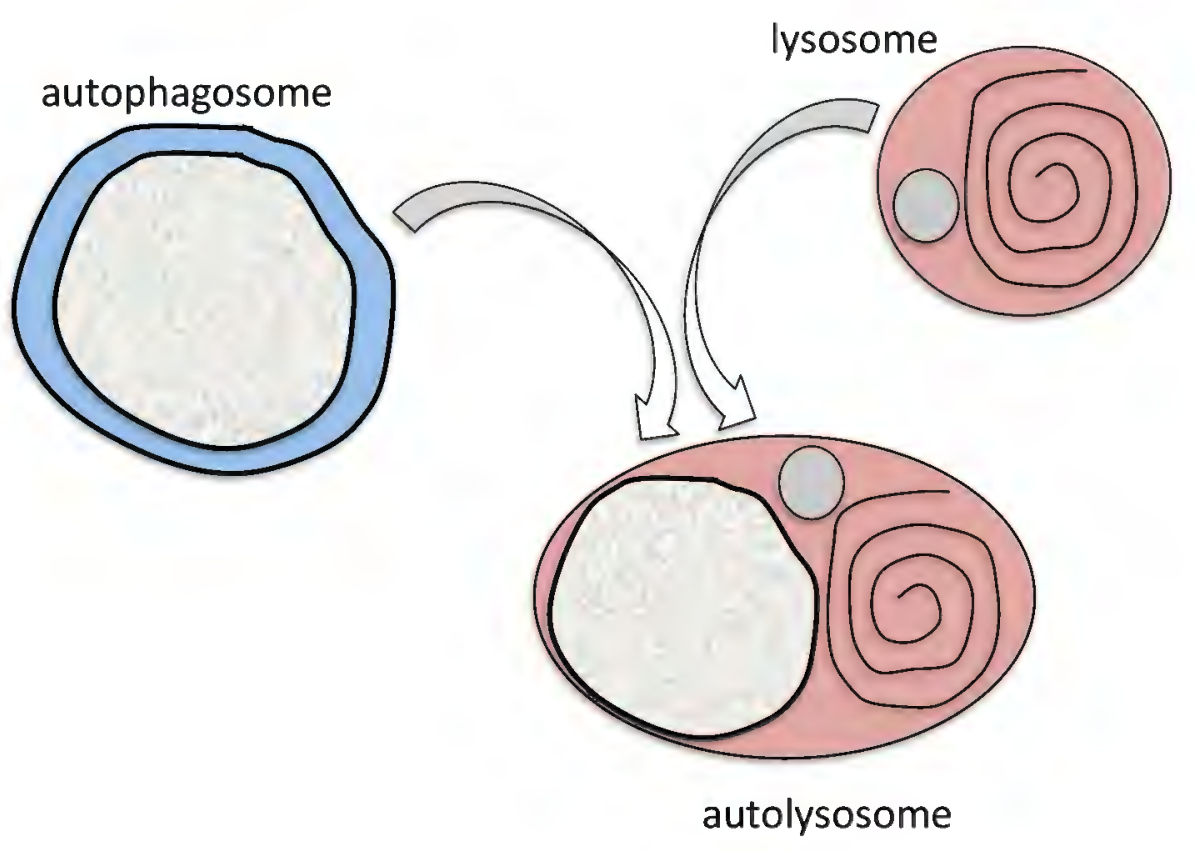

Figure 2 

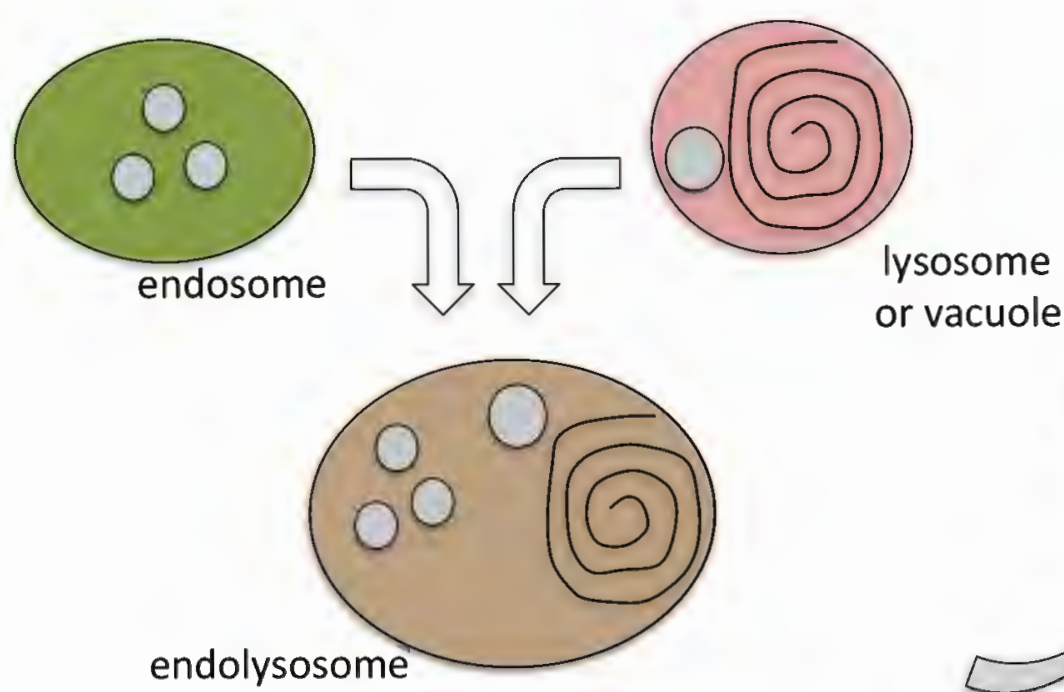

reformation of lysosome

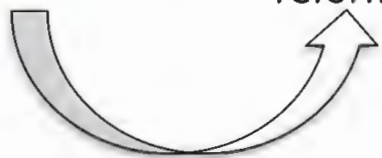

Figure 3 


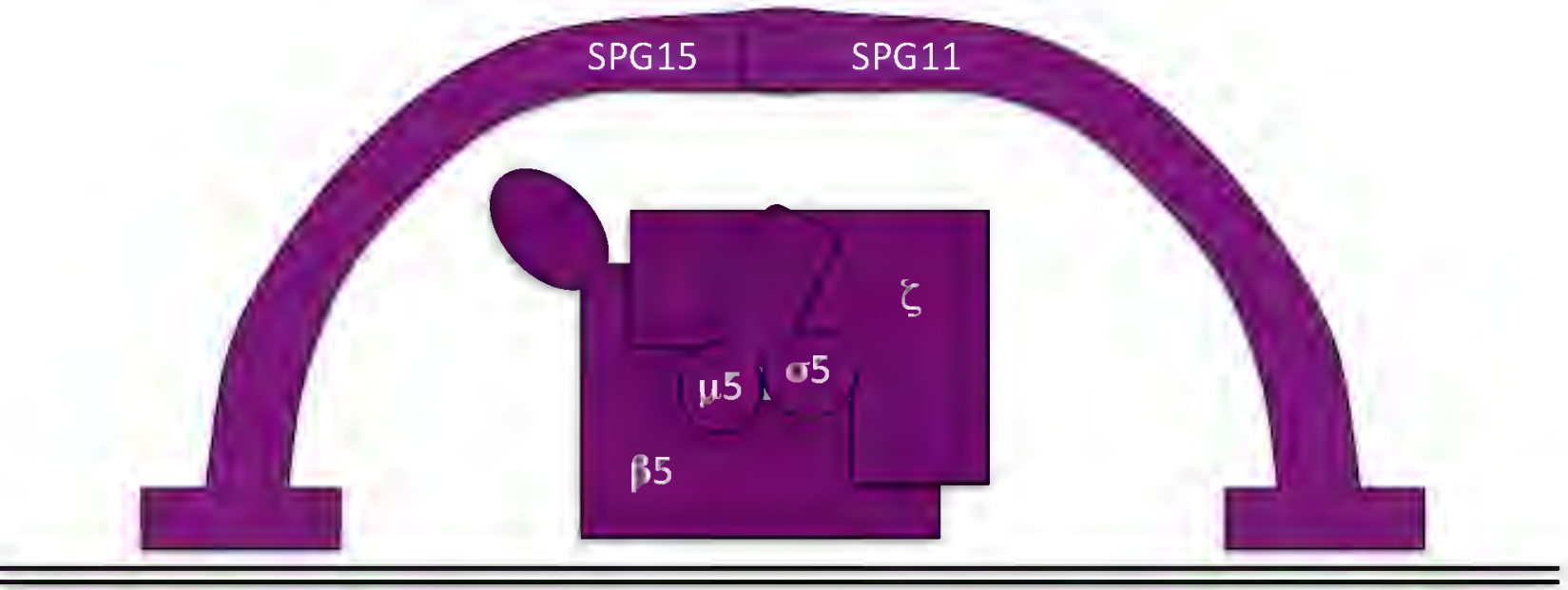

endolysosomal membrane

Figure 4 


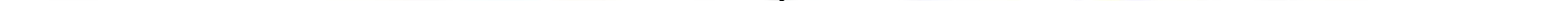

